\title{
Cognitive dysfunction as a major determinant of disability in patients with heart failure: results from a multicentre survey
}

\author{
G Zuccalà, G Onder, C Pedone, A Cocchi, L Carosella, C Cattel, P U Carbonin,
} $\mathrm{R}$ Bernabei, on behalf of the GIFA (SIGG-ONLUS) investigators

\begin{abstract}
Cognitive dysfunction is a frequent finding among older patients with left ventricular systolic dysfunction; however, the clinical outcomes of such a finding are unknown. Also, disability is a common condition in heart failure, poorly responding to commonly used cardiovascular medications.
\end{abstract}

The association between cognitive dysfunction and disability was assessed in 1583 patients with heart failure, but without cerebrovascular disease, previous stroke, or Alzheimer's disease, who were enrolled during 2 years of a multicentre pharmacoepidemiology survey. The association between groups of variables (demographics, comorbid conditions, medications, and objective tests, including the Hodkinson abbreviated mental test) and functional disability (as indicated by need for intensive assistance in at least one of Katz' activities of daily living) was first analysed using separate age and sex adjusted logistic regression models. Those variables, significant at a $p<0.1$ level in these models, were simultaneously entered into an age and sex adjusted summary regression model. Among 1583 patients suitable for analysis, cognitive dysfunction (as detected by abbreviated mental test score <7) was detected in $265 / 461$ disabled patients, and in $150 / 1122$ independent subjects $(p<0.0001)$. According to logistic regression analysis, cognitive dysfunction was associated with disability $(\mathrm{OR}=6.49 ; 95 \% \mathrm{CI}=4.39-9.59)$ after adjusting for potential confounders. Thus, cognitive dysfunction in patients with heart failure is independently associated with disability, which currently represents an overwhelming medical and financial problem to patients, caregivers, and public health services. As early recognition and treatment of low cardiac output states might reverse cognitive dysfunction, cost effective treatment for heart failure should include systematic diagnostic and therapeutic approaches to cognitive dysfunction.
(F Neurol Neurosurg Psychiatry 2001;70:109-112)

Keywords: dementia; heart failure; disability

Heart failure is the leading cause of admission to hospital in adults older than 65 . In the United States, rates of hospital admission for heart failure increased by $25 \%$ between 1985 and 1995 among patients older than 85; due to progressive aging of populations, the number of patients with heart failure will double within the next 40 years. ${ }^{1}$ Indeed, heart failure has already emerged as a major public health concern, mainly because this condition is associated with excess prevalence of dependence for the activities of daily living, such as walking, dressing, bathing, using the toilet, or eating. ${ }^{12}$ In fact, among older patients with heart failure disability strongly affects both quality of life and resource consumption. ${ }^{1-3}$ Accordingly, heart failure currently represents the single most costly cardiovascular illness in the United States. ${ }^{4}$ In addition, disability represents an independent risk factor for increased mortality and hospital admission in patients with left ventricular dysfunction. ${ }^{5}$

However, the determinants of disability in patients with heart failure are still unclear; for instance, reduced exercise capacity does not predict dependency for the activities of daily living among these subjects. ${ }^{6}$

Recently, heart failure has been associated with increased prevalence of cognitive dysfunction in older populations ${ }^{78}$; in these patients, cognitive dysfunction correlates with the degree of left ventricular dysfunction. ${ }^{7}$ Noticeably, as indicated by studies on cardiac transplantation or pacemaker implantation, timely correction of low output states in patients with heart failure can improve cognitive function. ${ }^{9}{ }^{10}$ However, the clinical consequences of cognitive impairment in subjects with heart failure are currently unknown.

In older populations, even moderate cognitive impairment is associated with increased prevalence of disability and need for institutional care. ${ }^{11}{ }^{12}$ We analysed the association between cognitive dysfunction and disability in 1583 elderly subjects admitted to hospital with 
heart failure who were enrolled in a multicentre pharmacoepidemiology study.

\section{Materials and methods}

We used the database of the Gruppo Italiano di Farmacoepidemiologia nell' Anziano (GIFA), a collaborative pharmacoepidemiology hospital study. The methods of the GIFA have been described in detail elsewhere. ${ }^{13}$ Briefly, all 8376 patients admitted to 81 academic centres throughout Italy from 1 May to 30 June and 1 September to 31 October, 1995, and 1997, were enrolled and followed up until discharge. For each patient a questionnaire was completed on admission and updated daily by a study physician who received specific training. Data were recorded using dedicated software ${ }^{14}$; the variables recorded included demographic characteristics, objective tests and measures, drugs taken before admission, during hospital stay, and at discharge, and admission and discharge diagnoses. In particular, we considered the hospital discharge diagnosis of congestive heart failure, which was verified by the study researchers. The accuracy of such a diagnosis could be tested in the 1993 GIFA survey against a "definite" diagnosis based on a validated series of physical and laboratory data. ${ }^{15}$ Using a score $\geqslant 8$ as reference diagnosis, ${ }^{16}$ the clinical diagnosis of congestive heart failure among 1687 subjects yielded $95 \%$ specificity (1123 true negatives, and 59 false positives). Cognitive performance was assessed using the Hodkinson abbreviated mental test (AMT).${ }^{17}$ This test has been proved reliable for detecting both mild cognitive impairment and dementia in older populations, ${ }^{18}$ and has been adopted in epidemiological surveys. ${ }^{19}$ Also, this test has been validated in Italian populations. ${ }^{20}$ Disability was defined as need for intensive assistance in at least one of Katz' activities of daily living. ${ }^{21}$ For patients with cognitive dysfunction, data on functional ability were collected from their caregivers. ${ }^{22}$ This might introduce some form of report bias, as cognitively intact patients often give higher estimates of their own functional ability compared with caregivers. Nevertheless, use of objective activities of daily living as reported by caregivers is widely recommended when assessing the functional ability of demented patients. $^{22-24}$

Drugs were coded according to the anatomical therapeutic and chemical codes. ${ }^{14}$ Diagnoses were coded according to the International Classification of Diseases, ninth edition, clinical modification codes. ${ }^{25}$ Comorbidity was quantified using the Charlson comorbidity index score by adding scores assigned to specific discharge diagnoses. ${ }^{26}{ }^{27} \mathrm{~A}$ score of 1 was attributed to myocardial infarction (codes 410-410.9, 412), congestive heart failure (codes 428-428.9), peripheral vascular disease $(443.9,441-441.9,785.4, \mathrm{~V} 43.4)$, cerebrovascular disease (430-438), dementia (290-290.9), chronic pulmonary disease (490496, 500-505, 506.4), rheumatological disease (710.0, 710.1, 710.4, 714.0-714.2, 714.8, 725), peptic disease (531-534.9, 531.4-531.7, 532.4-532.7, 533.4-533.7, 534.4-534.7), mild liver disease (571.2, 571.4-571.6), and diabetes (250-250.3, 250.7). The following conditions scored 2: diabetes with chronic complications (codes 250.4-250.6), hemiplegia, or paraplegia $(344.1,342-342.9)$, renal disease (582-582.9, 583-583.7, 585, 586, 588-588.9), any malignancy, including leukaemia and lymphoma (140-172.9, 174-195.8, 200-208.9). Moderate to severe liver disease (codes 572.2-572.8, 456.0-456.2) scored 3. Finally, a score of 6 was assigned to metastatic solid tumour (codes 196-199.1) and AIDS (042-044.9). In addition, coronary disease (codes 410-414.9), hypertension (401-405.9), heart failure (428-428.9), chronic pulmonary disease (491-496), diabetes (250-250.7), renal disease (582-582.9, 583-583.7, 585, 586, 588-588.9), and atrial fibrillation (4727.3) were analysed as separate variables. Patients with diagnoses of cerebrovascular disease or stroke (342-342.9, 430-439, 437.1) or Alzheimer's disease (290.1) were excluded from analyses.

Data of continuous variables are presented as mean values (SE). Statistical analyses were performed using SPSS for Windows 9.0 software; differences were considered significant at the $\mathrm{p}<0.05$ level. Analysis of continuous variables in relation to an AMT score $<7$ before hospital discharge was performed by analysis of variance (ANOVA) comparisons. $\chi^{2}$ analysis was used for dichotomous variables. Logistic regression analysis was used to estimate the association of variables of interest with disability. To assess the independent risk factors for disability which might confound the association of cognitive dysfunction with dependence for ADLs, groups of variables were examined in separate age and sex adjusted regression models with simultaneous introduction of covariates, as depicted in the table. ${ }^{78-31}$ These partial regression models included demographics (age, sex); comorbid conditions (coronary disease, hypertension, diabetes, renal disease, chronic pulmonary disease, atrial fibrillation, and the comorbidity score index); medications (use of calcium antagonists, digitalis, diuretics, ACE inhibitors, nitrates, antiplatelets); and objective tests (presence of cognitive dysfunction, serum sodium, potassium, and creatinine concentrations, and systolic blood pressure). Those variables, significant at the $p<0.1$ level in these initial models, were simultaneoulsy entered into a summary age and sex adjusted regression model having disability as the dependent variable. ${ }^{28-31}$

\section{Results}

Among those suitable for analyses, cognitive dysfunction was detected in $415 / 1583$ patients with heart failure, and in 2266/12052 control subjects; disability was found in $461 / 1583$ participants with heart failure, and in 2623/12052 control patients (Fisher's exact test $\mathrm{p}<0.0001$ for both comparisons).

The main characteristics of participants with heart failure according to presence of functional impairment are depicted in the left columns in the table. Among 1583 patients with heart failure who were suitable for analy- 


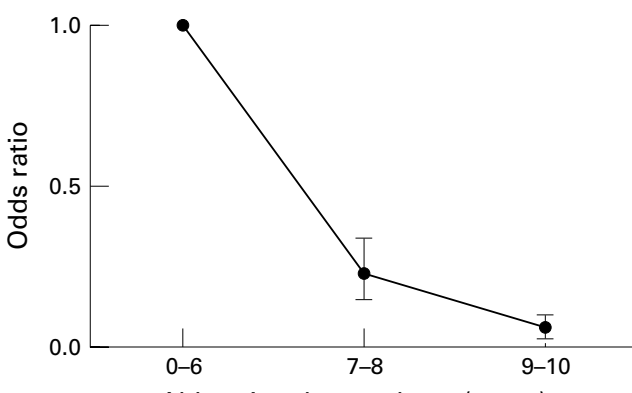

Abbreviated mental test (score)

Probability of disability among participants with heart failure according to their abbreviated mental test score levels ${ }^{17}$ as calculated by logistic regression analysis.

ses, cognitive dysfunction was detected in 265/ 461 disabled patients, and in 150/1122 independent subjects (Fisher's exact test $\mathrm{p}<0.0001)$.

According to unadjusted regression analysis (which only assessed the association between levels of cognitive dysfunction and disability), increasing AMT score was associated with decreasing probability of disability (figure). In the initial age and sex adjusted regression models, age, sex, diagnosis of coronary disease, hypertension, and chronic pulmonary disease, the comorbidity score index, cognitive dysfunction, systolic blood pressure, and use of ACE inhibitors were associated with disability at a $\mathrm{p}<0.1$ level (table).

When these variables were entered in the summary regression model, cognitive dysfunction was still associated with dependency in activities of daily living ( $\mathrm{OR}=6.49 ; 95 \% \mathrm{CI}$ 4.39-9.59).

Results of this regression model for the association between cognitive dysfunction and disability were almost unchanged $(\mathrm{OR}=6.63$; 95\% CI 4.41-9.97) when patients with systolic blood pressure $\geqslant 130 \mathrm{~mm} \mathrm{Hg}$ were considered.

Main characteristics of patients according to dependency in one or more activities of daily living, ${ }^{21}$ and odds ratios (OR) with 95\% confidence intervals (95\% CIs) in separate age and sex adjusted logistic regression models (including demographics, comorbid conditions, medications, and objective tests) having disability as dependent variable. The variables were simultaneously entered into the separate regression models

\begin{tabular}{|c|c|c|c|c|}
\hline & $\begin{array}{l}\text { Independent } \\
(n=1122) n[\%] \\
\text { or mean (SE) }\end{array}$ & $\begin{array}{l}\text { Disabled } \\
(n=461) n \\
{[\%] \text { or mean }} \\
(S E)\end{array}$ & $O R$ & $95 \% C I$ \\
\hline \multicolumn{5}{|l|}{ Demographics: } \\
\hline Age $(y)$ & 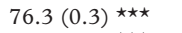 & $81.7(0.4)$ & 1.07 & $1.06-1.09^{\star \star \star}$ \\
\hline Sex (male) & $564[50.3]^{\star \star \star}$ & $183[39.7]$ & 0.83 & $0.66-1.05$ \\
\hline \multicolumn{5}{|l|}{ Comorbid conditions: } \\
\hline Coronary disease & $333[29.7]^{\star}$ & $111[24.1]$ & 0.67 & $0.57-0.95^{\star}$ \\
\hline Hypertension & $325[29.0]^{\star \star \star}$ & $87[18.9]$ & 0.57 & $0.43-0.76^{\star \star \star}$ \\
\hline Diabetes & $223[19.9]$ & $92[20.0]$ & 0.99 & $0.73-1.35$ \\
\hline Renal disease & $96[8.6]^{\star \star}$ & $60[13.0]$ & 1.15 & $0.76-1.74$ \\
\hline Chronic pulmonary disease & $219[19.5]$ & $79[17.1]$ & 0.71 & $0.52-0.98^{\star}$ \\
\hline Atrial fibrillation & $274[24.4]$ & $110[23.9]$ & 0.94 & $0.72-1.24$ \\
\hline Comorbidity score index ${ }^{26}$ & $1.97(0.04)^{\star \star \star}$ & $2.29(0.08)$ & 1.26 & $1.15-1.38^{\star}$ \\
\hline \multicolumn{5}{|l|}{ Medications: } \\
\hline Calcium antagonists & $303[27.0]^{\star}$ & $100[21.7]$ & 0.80 & $0.61-1.06$ \\
\hline Digitalis & $833[74.2]$ & $342[74.2]$ & 1.03 & $0.78-1.35$ \\
\hline Diuretics & $934[83.2]$ & $374[81.1]$ & 0.91 & $0.67-1.25$ \\
\hline ACE inhibitors & $455[40.6]^{\star \star \star}$ & $123[26.7]$ & 0.57 & $0.44-0.72^{\star \star \star}$ \\
\hline Nitrates & $583[52.0]^{\star}$ & $213[46.2]$ & 0.86 & $0.68-1.08$ \\
\hline Antiaggregants & $134[11.9]$ & $40[8.7]$ & 0.71 & $0.48-1.04$ \\
\hline \multicolumn{5}{|l|}{ Objective tests: } \\
\hline Cognitive dysfunction & $150[13.4]^{\star \star \star}$ & $265[57.5]$ & 6.51 & $3.80-11.15^{\star \star \star}$ \\
\hline Serum sodium (mEq/l) & $139.8(0.15)^{\star \star}$ & $138.8(0.31)$ & 0.95 & $0.90-1.01$ \\
\hline Serum potassium (mEq/l) & $4.3(0.02)$ & $4.3(0.04)$ & 0.66 & $0.43-1.01$ \\
\hline Serum creatinine $(\mathrm{mg} / \mathrm{dl})$ & $1.33(0.03)^{\star \star}$ & $1.47(0.05)$ & 1.21 & $0.93-1.58$ \\
\hline Systolic blood pressure ( $\mathrm{mm} \mathrm{Hg}$ ) & $137.0(0.76)^{\star \star \star}$ & $130.0(1.36)$ & 0.98 & $0.97-0.99^{\star \star}$ \\
\hline
\end{tabular}

${ }^{\star} \mathrm{p}<0.05 ;{ }^{\star \star} \mathrm{p}<0.01 ;{ }^{\star \star \star} \mathrm{p}<0.0001$ ( $v$ disabled in univariate analyses).
In addition, analysis of the interaction term by logistic regression modelling confirmed that the association between cognitive dysfunction and disability did not vary across blood pressure levels. Cognitive dysfunction was associated with the outcome $(\mathrm{OR}=7.49 ; 95 \%$ CI 5.05-11.12) also when serum sodium concentrations were entered into the summary regression model. Cognitive dysfunction was associated with disability (OR=8.69; $95 \% \mathrm{CI}$ 4.94-15.31) among patients with normal serum sodium (135-145 mEq/l); again, analysis of the interaction term did not evidence any variations in the association between cognitive dysfunction and disability according to serum sodium concentrations.

\section{Discussion}

Results of the present study indicate that among patients with heart failure cognitive dysfunction is associated with increased probability of disability, independently of any potential confounders. In particular, the association between cognitive dysfunction and disability is not influenced by age, sex, comorbidity, or medications. In addition, low blood pressure concentrations and hyponatraemia, which are common features of advanced heart failure, do not affect the relation between cognitive dysfunction and disability.

Most recently, an abnormal prevalence of cognitive impairment (ranging from 53\% to $58 \%$ ) has been demonstrated in older populations with heart failure. ${ }^{78}$ This figure is impressive, when considering the estimated prevalence of heart failure (4.9 million in the United States) among western populations. ${ }^{4}$ Cognitive dysfunction in these patients correlates inversely with reduced left ventricular systolic function. ${ }^{7}$ Indeed, both CT and neuropathological studies have evidenced white matter lesions in older demented patients with heart failure and systolic hypotension. ${ }^{32} 33$ Cerebral ischaemia represents another potential source of cerebral damage among subjects with heart failure, even though patients with cerebrovascular disease or stroke had been excluded from our analyses; in addition, the incidence of cerebral embolism among such patients is low. ${ }^{34}$ However, no data are currently available showing the possible impact of cognitive dysfunction on the clinical conditions of patients with chronic heart failure.

On the other hand, disability is a highly prevalent, yet unexplored condition among subjects with heart failure. ${ }^{12}$ Loss of independence for activities of daily living in these subjects is not related to exercise capacity; furthermore, it does not respond to treatment with vasodilating agents, which is otherwise acknowledged as "effective" ${ }^{65}$ However, disability is a powerful predictor of increased mortality and rates of hospital admission among patients with heart failure. ${ }^{5}{ }^{6}$ In addition, dependency for the activities of daily living yields a deep impact on patients' quality of life, and is associated with marked raises in both direct and indirect costs related to the management of heart failure. ${ }^{3}$ For instance, 
about $20 \%$ of patients admitted to hospital with heart failure have to be discharged to long term care. ${ }^{1}$

Assessing the determinants of disability among older patients is a difficult task, as when adjusting for potential confounders it is necessary to consider several variables, which imposes the use of complex modelling. The role of confounders in this setting is highlighted by those variables (such as use of calcium antagonists or nitrates) whose association with disability could not be further evidenced in the present study after adjusting for age, sex, and concurrent medications (table). Indeed, even "systematic" statistical adjusting cannot definitely rule out the effects of confounding or bias, which is the main limitation of observational studies. However, use of high quality clinical databases yields the highest potential for studies on complex clinical issues, so allowing for the improvement the quality of health care. ${ }^{36}$

As suggested by prospective studies in patients who underwent cardiac transplantation or pacemaker implantation, cognitive dysfunction in subjects with heart failure might be reversible, at least before the development of cerebral alterations due to prolonged hypoperfusion. ${ }^{9} 10$

At present, several strategies for multidisciplinary treatment for heart failure are under evaluation. ${ }^{3}$ Our data indicate that systematic neuropsychological assessment of older patients with heart failure for prompt recognition of cognitive dysfunction and correction of low output states should be assessed in trials aiming at reducing the burden of disability in these subjects.

The GIFA study was partially supported by a grant from the National Research Council (No 94000402) and by Neopharmed.

1 Haldeman GA, Croft JB, Giles WH, et al. Hospitalization of patients with heart failure: national hospital discharge survey, 1985-95. Am Heart f 1999;137:352-60.

2 Wolinsky FD, Smith DM, Stump TE, et al. The sequelae of hospitalization for congestive heart failure among older adults. F Am Geriatr Soc 1997;45:558-63.

3 Rich MW, Nease RF. Cost-effectiveness analysis in clinica practice. The case of heart failure. Arch Intern Med 1999;159:1690-700

4 American Heart Association. 1998 Heart and stroke statistical update. Dallas TX, USA: AHA, 1998.

5 Konstam V, Salem D, Pouleur H, et al. Baseline quality of life as a predictor of mortality and hospitalization in 5025 patients with congestive heart failure. SOLVD investigapatients with congestive heart failure. SOLVD investiga-
tions. Studies of left ventricular dysfunction investigators. Am $\mathcal{F}$ Cardiol $1996 ; 78: 890-5$.

6 Walsh JT, Charlesworth A, Andrews R, et al. Relation of daily activity levels in patients with chronic heart failure to daily activity levels in patients with chronic heart fail
long-term prognosis. Am f Cardiol 1997;15:1364-9.

7 Zuccalà G, Cattel C, Manes-Gravina E, et al. Left ventricular dysfunction: a clue to cognitive impairment in older patients with heart failure. F Neurol Neurosurg Psychiatry 1997;63:509-12.

8 Cacciatore F, Abete P, Ferrara N, et al. Congestive heart failure and cognitive impairment in an older population. $f$ Am Geriatr Soc 1998;46:1343-8.

9 Scahll RR, Petrucci RJ, Brozena SC, et al. Cognitive function in patients with symptomatic dilated cardiomyopathy before and after cardiac transplantation. $\mathcal{J} \mathrm{Am}$ Coll Cardiol 1989;14:1666-72.
10 Koide H, Kobayashi S, Kitani M, et al. Improvement of cerebral blood flow and cognitive function following pacemaker implantation in patients with bradycardia. Gerontology 1994;40:79-85.

11 Zhu L, Fratiglioni L, Zhenchao G, et al. Association of stroke with dementia, cognitive impairment, and functional disability in the very old. Stroke 1998;29:2094-9.

12 Graham JE, Rockwood K, Beattie BL, et al. Prevalence and severity of cognitive impairment with and without dementia in an elderly population. Lancet 1997;349:1793-6.

13 Pahor M, Manto A, Pedone C, et al. Age and severe adverse drug reactions caused by nifedipine and verapamil. $\mathcal{F}$ Clin Epidemiol 1996;49:921-8

14 Pahor M, Chrischilles EA, Guralnik JM, et al. Drug data coding and analysis in epidemiologic studies. Eur $\mathcal{F}$ Clin Epidemiol 1994;10:405-11.

15 Antonelli Incalzi R, Pedone C, Pahor M, et al. Reason prompting digitalis therapy in the acute care hospital. $f$ Gerontol Med Sci, (in press).

16 Carlson KJ, Lee DC, Goroll AH, et al. An analysis of physicians' reasons for prescribing long-term digitalis therapy in out-patients. F Chron Dis 1985;38:733-9.

17 Hodkinson HM. Evaluation of a mental test score for assessment of mental impairment in the elderly. Age Ageing 1972;1:233-8.

18 Gomez de Caso JA, Rodriguez-Artalejo F, Claveria LE, et al. Value of Hodkinson's test for detecting dementia and mild cognitive impairment in epidemiological surveys. Neuroepidemiology 1994;13:64-8.

19 Gale CR, Martyn CN, Cooper C. Cognitive impairment and mortality in a cohort of elderly people. BMF 1996;12: 608-11.

20 Rocca WA, Bonaiuto S, Lippi A, et al. Validation of the Hodkinson's abbreviated mental test as a screening instrument for dementia in an Italian population. Neuroepidemiology 1992;11:288-95.

21 Katz S, Ford AB, Moskowitz RW, et al. Studies of illness in the aged: the index of ADL: a standardized measure of biological and psychosocial function. $7 A M A$ 1963: 185:41419.

22 Ostbye T, Tyas S, McDowell I, et al. Reported activities of daily living: agreement between elderly subjects with and without dementia and their caregivers. Age Ageing 1997;26: 99-106.

23 Gelinas I, Gauthier L, McIntyre M, et al. Development of a functional measure for persons with Alzheimer's disease: the disability assessment for dementia. Am $\mathcal{F}$ Occup Ther 1999;53:471-81.

24 Albert SM, Del Castillo-Castaneda C, Sano M, et al. Quality of life in patients with Alzheimer's disease as reported by patient proxies. F Am Geriatr Soc 1996;44:1342-7.

25 Public Health Service-Health Care Financing Administration. International classification of diseases, 9 th rev. Washington, DC: PHS-HCFA, 1980

26 Charlson ME, Pompei P, Ales KL, et al. A new method of classifying prognostic comorbidity in longitudinal studies: development and validation. $\mathcal{F}$ Chron Dis 1987;40:373-83.

27 Deyo RA, Cherkin DC, Ciol MA. Adapting a clinical comorbidity index for use with ICD-9-CM administrative databases. F Clin Epidemiol 1992;45:613-19.

28 Pahor M, Guralnik JM, Ferrucci L, et al. Calcium-channel blockade and incidence of cancer in aged populations. Lancet 1996;348:493-7.

29 Pahor M, Guralnik JM, Furberg CD, et al. Risk of gastrointestinal haemorrhage with calcium antagonists in hypertensive persons over 67 years old. Lancet 1996;347: 1061-5.

30 Zuccalà G, Pedone C, Cocchi A, et al. Use of calcium antagonists and haemoglobin loss in hospitalised elderly: cohort study. Clin Pharmacol Ther 2000;67:314-22.

31 Zuccalà G, Pahor M, Landi F, et al. Use of calcium antagonists and need for perioperative transfusion in older patients with hip fracture: observational study. BMF 1997; 314:643-4.

32 Tarvonen-Schroder S, Roytta M, Raiha I, et al. Clinical features of leuko-araiosis. F Neurol Neurosurg Psychiatry 1996; 60:431-6.

33 Brun A, Englund E. A white matter disorder in dementia of the Alzheimer type: a pathoanatomical study. Ann Neurol 1986;19:253-62.

34 Katz SD, Marantz PR, Biasucci L, et al. Low incidence of stroke in ambulatory patients with hearth failure: a prospective study. Am Heart f 1993;126:141-6.

35 Walsh JT, Andrews R, Evans A, et al. Failure of effective treatment for heart failure to improve normal customary activity. Br Heart $\mathcal{F}$ 1995; 74:373-6.

36 Black N. High-quality clinical databases: breaking down barriers. Lancet 1999;353:1205-6. 